\title{
On the Role of the Plasma Composition in the Magnetic Field Evolution in Plasma Opening Switches
}

\author{
D. Osin, R. Doron, R. Arad, K. Tsigutkin, A. Starobinets, V. Bernshtam, A. Fisher, A. Fruchtman, Y. Maron, \\ and A. Tauschwitz
}

\begin{abstract}
High spatial- and temporal-resolution spectroscopic methods are employed to perform detailed studies of the interaction between the propagating magnetic field and a multi-ion-species plasma. The experiment is performed in a plasma-opening-switch configuration, in which a 150 -kA current of $\sim 400 \mathrm{~ns}$ duration is conducted through a plasma. Recent observations demonstrated a new phenomenon of simultaneous rapid magnetic field penetration into the heavy-ion plasma and specular reflection of the light-ion plasma, leading to ion-species separation. Additionally, noticeable inconsistencies between experimental results and theories were found. The current paper summarizes these recent results and discusses the aspect of the role of the plasma composition in the magnetic field evolution and ion dynamics. In order to systematically investigate the effect of the plasma composition, a method for producing plasmas with controllable compositions, based on spatial species separation and electrode heating, is presented. This method allows for achieving plasmas with varying proton-to-carbon ion ratios, however, at different electron densities. Measurements are described for studying the relation between the magnetic field propagation velocity and the plasma composition and density; however, this relation is not yet satisfactorily clear.
\end{abstract}

Index Terms-Magnetic field-plasma interaction, multi-ion species plasma, plasma devices, plasma spectroscopy.

\section{INTRODUCTION}

$\mathbf{P}$ LASMA opening switch (POS) can be used to switch multi-MA currents with typical rise times of 0.1 to $1 \mu$ s to a load within tens of nanoseconds. In such a system, a pulsed current is conducted through a plasma bridge between two electrodes and is then switched rapidly to a load due to a fast rise in

Manuscript received September 29, 2003; revised May 13, 2004. This work was supported in part by the German-Israeli Project Cooperation Foundation (DIP), the Minerva Foundation, Munich, Germany, the Israeli Science Foundation (ISF), and Sandia National Laboratories, Sandia, NM.

D. Osin, R. Doron, K. Tsigutkin, A. Starobinets, V. Bernstam, and Y. Maron are with the Faculty of Physics, Weizmann Institute of Science, Rehovot 76100, Israel (e-mail: dimaosin@weizmann.ac.il; rdoron@weizmann.ac.il; k.tsigutkin@weizmann.ac.il; nets@weizmann.ac.il; vladimir.bernshtam@weizmann.ac.il; Fnmaron@wisemail.weizmann.ac.il; casper@plasmagate.weizmann.ac.il).

R. Arad is with Sorec Nuclear Research Center, Yavne 81800, Israel (e-mail: fnarad@yahoo.com).

A. Fisher is with the Faculty of Physics, Technion-Israel Institute of Technology, Haifa 32000, Israel (e-mail: amfisher@ technion.il).

A. Fruchtman is with the Holon Academic Institute of Technology, Holon 58102, Israel (e-mail: fnfrucht@hait.ac.il).

A. Tauschwitz is with Gesellschaft für Schwerionenforschung, GSI Darmstadt, D-64291, Darmstadt, Germany (e-mail: A.Tauschwits@dsi.de).

Digital Object Identifier 10.1109/TPS.2004.835941 the plasma resistance. The POS has been applied at the 0.5 to 4 MA current levels to drive electron-beam diodes [1]-[3], [30] and z-pinches [4], [5], [31]. Howeve r, problems related to the efficiency of energy coupling to loads prohibited a wide use of the technique. Experiments have shown that the current transfer to the load is often accompanied by plasma reaching the load [6]-[10]. Furthermore, continued plasma presence in the A-K gap may limit the voltage generated on the load. Since the POS offers unique capabilities in pulsed-power applications, a large effort has been invested in improving its performance. It appears, however, that a thorough understanding of the physics processes that govern the behavior of plasmas under pulsed magnetic field is essential for controlling the operation of POSs. Such an understanding is also relevant to a wide range of astrophysical and laboratory plasmas.

The interaction between the plasma in pulsed-power systems and a pulsed magnetic field includes two basic competing phenomena: plasma pushing by the magnetic field and penetration of the magnetic field into the plasma. Early theories characterizing the plasma pushing by the $\vec{j} \times \vec{B}$ force and describing the magnetic field penetration by classical diffusion disagree with observations. Noticeably, observations have shown rapid magnetic field penetration much faster than predicted by classical diffusion based on the plasma resistivity [11]-[15]. Other theories [16]-[18] have shown that nonuniformity in the plasma, giving rise to a varying Hall electric field, may result in an enhanced magnetic field penetration. However, as yet, theories and simulations based on Hall field penetration fall short of predicting the details of the fast magnetic field penetration and the broad current channel measured in recent experiments [15].

In existing theories (based on diffusion, plasma pushing, and the Hall-effect), the plasma composition plays no direct role. In particular, the classical Hall theory addresses in detail the situation in which the velocity of the magnetic field propagation is larger than that of the pushed ions. Most of the dissipated magnetic field energy is then imparted to the electrons. A POS experiment of ideal single-species plasma is very difficult to realize. In fact, even if the plasma consists of predominantly single element, the ionization processes of the prefilled plasma, occurring due to the high-current application, result in different charge state ions (except for a pure proton plasma). Furthermore, the interaction of the plasma with the electrodes may result in a production of secondary plasma that is different from the primary plasma [19]. It has been demonstrated in experiments [20]-[22], 
which stimulated simulations [23] that for plasmas of different compositions, magnetic field penetration and light ion reflection (protons) can occur simultaneously resulting in ion-species separation. This in turn, may affect the plasma dynamics, switch opening and the energy coupling to the load.

The present report focuses on the role of the plasma composition in the evolution of pulsed magnetic fields in the plasma, which, to the best of our knowledge, is specifically here addressed for the first time. Based on previous and current observations of the ion dynamics in multispecies POS, the report includes a discussion that provides the motivation to investigate the role of the plasma composition. An essential first step in such an investigation should be the construction of a plasma source of a controllable composition. We report here on the development of a method that allows for obtaining plasma of an adjustable proton-to-carbon ion ratio. This method is implemented in a planar POS scheme and used to measure the magnetic field propagation velocity for plasmas of different compositions.

\section{EXPERIMENTAL SETUP AND DIAGNOSTICS}

In this study, we use a planar POS configuration depicted in Fig. 1. It consists of two 14-cm-wide electrodes separated by a $2.5-\mathrm{cm}$ gap. The current rises to $150 \mathrm{kA}$ in $400 \mathrm{~ns}$. A surface-flashover (flashboard) plasma source, driven by a single $2.8-\mu \mathrm{F}$ capacitor charged to $35 \mathrm{kV}$, is mounted above the anode. The flashboard plasma is mainly composed of protons and C III-V ions. Secondary plasma, produced due to the impinging flashboard-plasma on the electrodes, is mainly composed of $\mathrm{H}$, C II-III, and O II-III ions. Details of the flashboard-plasma parameters are given in [19]. In the 10-cm-long plasma prefilled region, the electrodes are of high (90\%) transparency in order to ensure free plasma flow through the anode and little plasma stagnation near the cathode. We define $x=0$ as the cathode surface, $y=0$ the center of the electrodes, and $z=0$ the generator-side edge of the transparent anode. The term "axial" is here used to denote the $z$-direction from the generator toward the load.

The high transparency of the electrodes increases the current density at the electrodes and may result in the ejection of material into the gap during the current conduction. However, previous studies have shown that this effect is insignificant [19]. Furthermore, to estimate the effect of the electrode-wire-geometry, several experiments were performed with electrodes covered by a stainless steel mesh. These experiments yielded similar results to those found without the mesh [15].

For the spectroscopic observations, light is collected by a 1-m UV-visible spectrometer equipped with a 2400 -groove $/ \mathrm{mm}$ grating. The output of the spectrometer can be attached either to an intensified CCD (ICCD) camera or to an array of photomultipliers (PMTs) via a bundle of optical fibers. Using the latter, the spectral resolution achieved is $0.07 \AA$. The use of the gated (5-ns) ICCD camera allows for recording broad wavelength band, and the PMT's provide a 7-ns temporal resolution for a narrower band. The spatial resolution in the plane perpendicular to the line of sight, determined by the spectrometer input slit and imaging optics, is $\sim 0.4 \times 2 \mathrm{~mm}$. Higher resolutions are impractical due to insufficient light intensities.

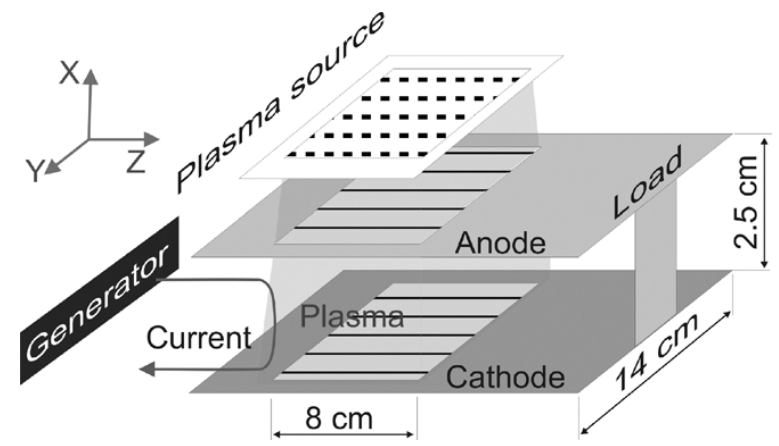

Fig. 1. Schematic description of the planar POS. The magnetic field propagates in the $z$-direction (i.e., from the generator side toward the load).

In order to obtain three-dimensional (3-D) spatially resolved measurements, we utilize our recently developed plasma-doping techniques. In this approach, the plasma is locally doped with atoms or ions, the line emission of which can be used for diagnosing the local plasma parameters (spectroscopic studies have been made to verify that the doped species densities are sufficiently low to cause no significant effect on the main-plasma properties [24]).

The spatial resolution along the line of sight is thus determined by the dopant column width, which is typically in the range $1-3 \mathrm{~cm}$. Two doping methods are used: a gas doping technique and a surface-flashover method (previously described in [25]). The dopant sources are mounted outside the A-K gap about $0.5 \mathrm{~cm}$ from the cathode, and the injection is in the $x$ direction.

\section{ION DyNAMICS IN MULTI-SPECIES PlASMAS}

As mentioned in Section I, previous measurements [20], [21] revealed the phenomenon of ion separation during the magnetic field penetration into a multispecies plasma in a short-duration experiment ( $\sim 100 \mathrm{~ns})$. The observations showed that the light-ion plasma (protons) is pushed ahead, whereas the heavy ion plasma lags behind the propagating magnetic field. Recent measurements performed in the present, long-duration $(\sim 400$ ns), planar POS configuration were used to gain more detailed information on the velocities of the various ion species. The axial ion velocities are determined from line Doppler shifts observed in the $z$ direction from the load side. Such measurements for carbon ions (a primary component of the plasma) give the axial velocity distribution integrated over the entire plasma column.

A clear picture of the evolution of the axial velocities can be obtained by means of the doping techniques described in Section II. In Fig. 2, we present an example of the line profile of He II dopant, injected in the middle of the plasma axial dimension, and measured at three different moments in time. Fig. 2 demonstrates ion acceleration toward the load, combined with a broadening of the velocity distribution (reflected by a broadened line profile). Other ions (except protons) that were injected into the plasma exhibited similar behavior. Based on previous measurements of the magnetic field propagation velocity [15], the present measurements also show that the onset of the axial acceleration of the ions, at different locations in the plasma, is correlated with the arrival of the propagating magnetic field. 


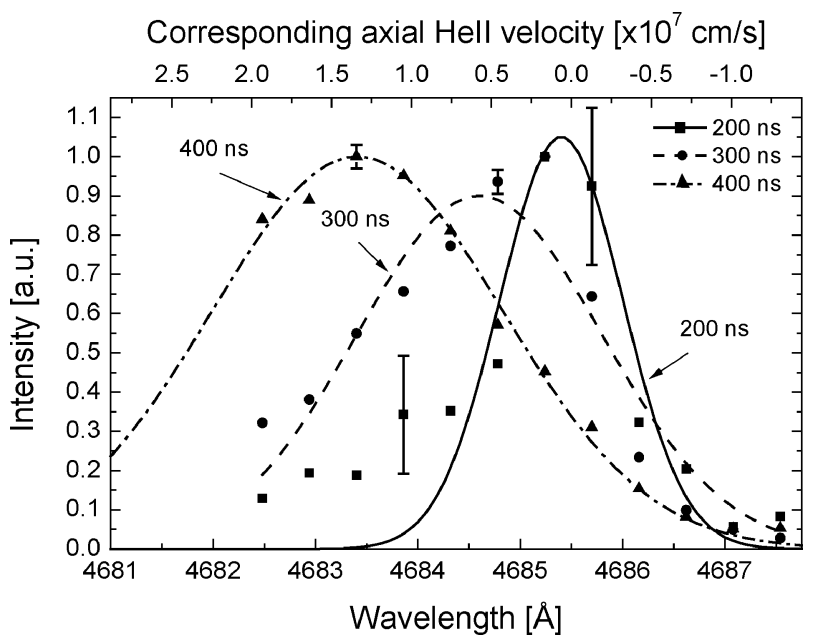

Fig. 2. He II $(n=4 \rightarrow n=3)$ spectral line profiles observed in the $z$-direction at $x=1.0$ and $y=0 \mathrm{~cm}$ for three different times $(200,300$, and $400 \mathrm{~ns}$ ) after the current generator application. In these experiments, helium was injected at $z=3.7 \mathrm{~cm}$.

In contrast to the heavier ions, measuring the proton velocity distribution is more difficult. In the present work, the proton axial velocities are obtained from charge-exchange spectroscopy [26], [32]. In this method, we make use of the gas-doping technique to produce a hydrogen-gas dopant column. This allows for charge-exchange processes between the accelerated protons and the dopant-hydrogen, making possible a reliable determination of the proton velocities using the Doppler shifted lines emitted from the fast hydrogen atoms. The mean measured axial velocity of the protons is found to be $\sim 7 \times 10^{7} \mathrm{~cm} \cdot \mathrm{s}^{-1}$, which is about twice the measured magnetic field velocity.

A complete report on the ion dynamics measurements, including a quantitative analysis of the results, is given in a subsequent paper [25]. Here, in Fig. 3, we summarize the results by presenting the peak axial velocities of the various ions, measured at the end of the current pulse, as a function of the charge to atomic mass ratio $(Z / m)$. The ion velocities are normalized to the magnetic field velocity. From Fig. 3, it is seen that there is a clear dependence between the velocity acquired by each ion species and the parameter $Z / m$. This behavior, particularly the behavior of the protons that acquire an average velocity of twice the magnetic field velocity, suggests that the fraction of the energy dissipated on the ions by the magnetic field strongly depends on the plasma composition.

The phenomenon of simultaneous magnetic field penetration into the heavier ion plasma and the reflection of the light-ion plasma is not predicted by available theories. In addition, some other observations are inconsistent with current theories. In a previous work, the measured magnetic field propagation velocity along the plasma column was found to be faster than predicted by diffusion [15]. In that experiment, the magnetic field spatial distribution was also found to disagree with diffusion. Higher field velocities are in principle possible via the Hall field mechanism due to electron density gradients [16]-[18]. However, experiments with reversed polarity had no significant effect on the magnetic field evolution, in contradiction to the predictions of the Hall theory.

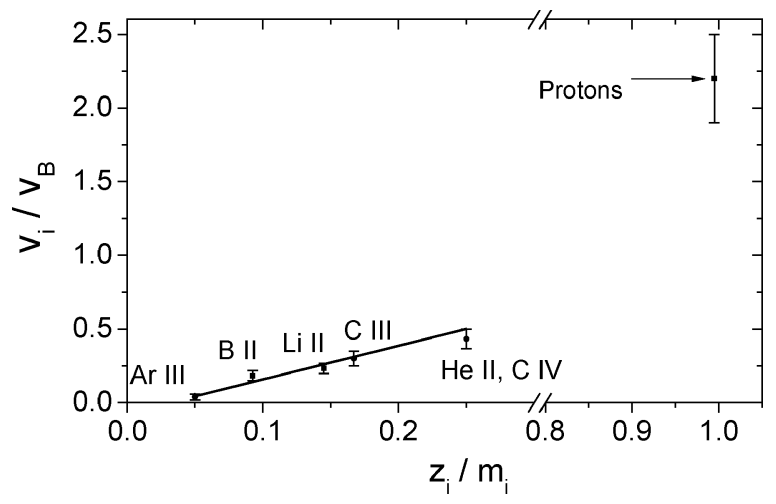

Fig. 3. Peak ion velocities in the $z$-direction normalized to the magnetic field front velocity $V_{B}$, as a function of the ion charge-to-mass ratio (the ion mass $m_{i}$ is given in proton-mass units) observed at $1 \mathrm{~cm}$ from the cathode in the middle of the plasma $y$-dimension. The proton and carbon ion velocities are integrated over the plasma length.

Recently, a possible mechanism for explaining these results based on the formation of small scale fluctuations was proposed [15]. In this explanation, the reflection of the light ions (within the heavier-ion plasma) could be susceptible to the growth of the Rayleigh-Taylor instability, leading to the formation of smallscale density fluctuations that enhance the Hall penetration. The enhancement of the Rayleigh-Taylor growth rate in the presence of the Hall term has been predicted theoretically [27] in the context of astrophysical plasmas. Since these density fluctuations are self-generated due to the light-ion (protons) pushing, they are expected to be nondependent on the current flow-direction (and therefore on the current polarity), which is consistent with our findings.

The observed ion dynamics imply an important role for the plasma composition in the processes that lead to the rapid magnetic field penetration and magnetic energy dissipation. Further investigations of the ion dynamics of multispecies plasma with controlled plasma composition may prove helpful for better understanding of the phenomena reported above and, consequently, for progress in switch opening and coupling to the load.

\section{Control of the Plasma Composition}

In order to systematically investigate the role of the plasma composition in the magnetic field evolution, it is desirable to develop procedures with controlled plasma compositions, particularly, with an adjustable fraction of protons and heavier ions. In the present work, we control the plasma composition by taking an advantage of the different times of flight of ions with different charge to mass ratios, ejected from the flashboard. In this method, we mount the flashboard plasma source $4.5 \mathrm{~cm}$ away from the anode (instead of $3 \mathrm{~cm}$ in the previous setup). Due to the larger distance, the faster protons reach the anode earlier than the carbon ions, filling the volume between the electrodes predominantly with proton plasma. The carbon ions reach the anode at later times, gradually changing the plasma composition at the A-K gap. Hence, the time delay between the flashboard discharge and the application of the generator current determines the plasma composition during the current flow and POS operation. 


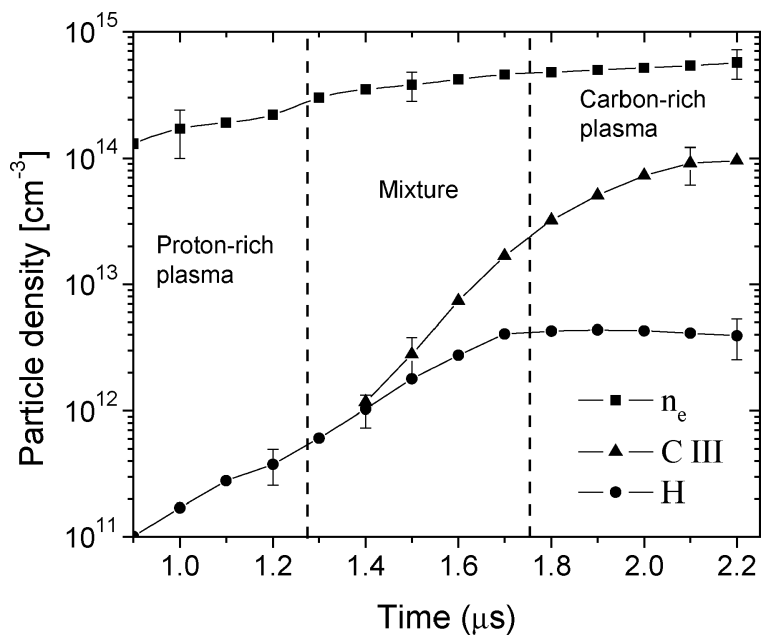

Fig. 4. Time evolution of the electron, hydrogen, and C III densities, observed $3 \mathrm{~mm}$ from the cathode with no generator current. The vertical dashed lines indicate a rough distinction between three ranges of plasma compositions. $t=0$ represents the discharge of the flashboard plasma source.

In order to determine the plasma composition in the A-K gap as a function of time, we have employed spectroscopic measurements combined with collisional-radiative calculations and use of data obtained in previous measurements. Time dependent measurements of the Stark broadening of $\mathrm{H}_{\alpha}$ and $\mathrm{H}_{\beta}$ are used to determine the electron density evolution [19]. The electron density inferred and the previously obtained temperature are then used to construct a collisional-radiative model to simulate the emission of the $\mathrm{H}_{\alpha}$ and the C III $2 \mathrm{p}^{2}{ }^{1} \mathrm{D}_{2}-2 \mathrm{~s} 2 \mathrm{p}{ }^{1} \mathrm{P}_{1}$ line at $2297 \AA$, allowing for the determination of the hydrogen and C III densities. The results of the electron, hydrogen, and C III densities as a function of time are presented in Fig. 4. The hydrogen density at early times (1-1.2 $\mu \mathrm{s})$ given in Fig. 4 reflects the arrival of fast protons to the interelectrode gap since these fast-hydrogen atoms are produced by charge-exchange processes between the protons accelerated (by the $\vec{j} \times \vec{B}$ force) from the flashboard and hydrogen atoms released from the flashboard surface. The measured electron and C III densities shown in Fig. 4 allow for the determination of the proton-to heavier ion (mostly carbon ions) ratio as a function of time. This is done by scaling the results for the abundance ratios among the heavier ions found in previous measurements [19] and the requirement for charge neutrality. For $t=1 \mu \mathrm{s}$, we obtain an electron density of $\sim 1 \times 10^{13} \mathrm{~cm}^{-3}$ contributed by the heavier ions (mostly C IV and $\mathrm{C} \mathrm{V}$ ), i.e., over $90 \%$ of the plasma are protons. The lack of data for C III density for short time delays $(t<1.4 \mu \mathrm{s})$ is due to the small number of carbon ions emitting hardly detectable amount of light. For $t=2 \mu \mathrm{s}$, we obtain that the heavier ions (mostly the slower C III and C IV) constitute over $95 \%$ of the plasma. The variation of the composition of the plasma filling the A-K gap occurs on a time scale of $\sim 1 \mu \mathrm{s}$, which is about three times longer than the POS current-conduction period, and therefore, the plasma composition is not entirely constant during the POS operation. Nevertheless, this method allows for operating the POS with selected effective mean compositions, ranging from $\sim 90 \%$ protons to over $95 \%$ carbon ions. A drawback of this method of controlling the plasma composition,

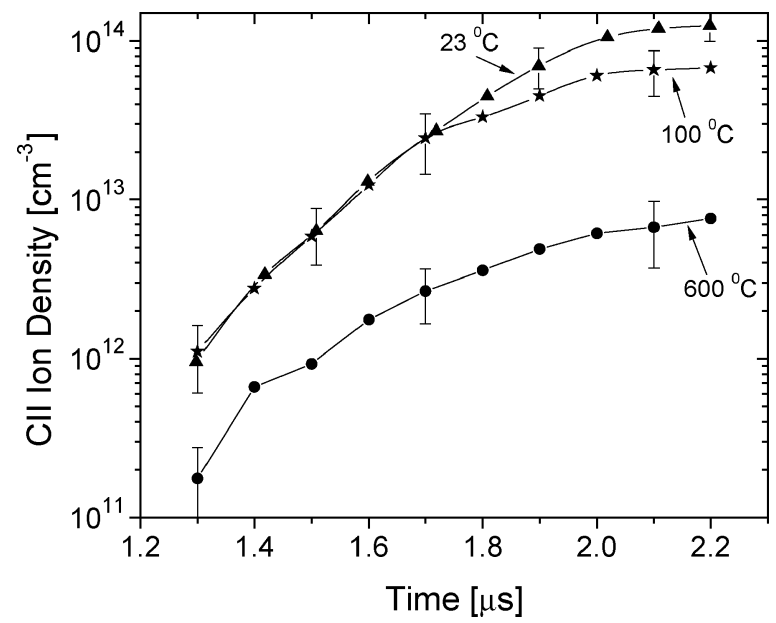

Fig. 5. Effect of electrode heating on the time evolution of C II observed 3 $\mathrm{mm}$ from the cathode with no generator current. $t=0$ represents the onset of the discharge of the flashboard plasma source.

as can be seen from Fig. 4, is that the temporal variation of the plasma composition is accompanied by variation of the plasma density. This difficulty will be addressed in a future work.

The control of the plasma composition can be further improved by minimizing the effects of the secondary plasma produced due to the electrode sputtering. We have investigated the effect of electrode cleaning by heating. The electrodes were heated up to $\sim 600^{\circ} \mathrm{C}$, and line intensities of the dominant secondary plasma constituents (H, C II, C III, O II) were observed. The observed line emission was translated into ion densities using collisional-radiative calculations based on the measured electron density and temperature. Measurements of the $\mathrm{C}$ II density, which were performed at various electrode temperatures, as a function of time are presented in Fig. 5. These measurements were performed at $3 \mathrm{~mm}$ from the cathode and $30 \mathrm{~min}$ after a steady electrode temperature was reached. It can be seen in Fig. 5 that the $\mathrm{C}$ II densities decrease with increasing temperature of the electrodes. At $600^{\circ} \mathrm{C}$, the C II densities are lower relative to those measured with electrodes at room temperature by a factor of $\sim 5$ for short time delays and by $\sim 15$ for longer time delays. This trend is due to two main reasons. As was previously shown [19], for short time delays, the relative portion of the secondary plasma, produced by electrode sputtering, is lower than that produced for longer time delays and, hence, cannot be significantly affected by the electrode heating. The other reason is that for longer time delays, the higher plasma density enhances the ionization of carbon atoms; thus, the observed effect of the electrode heating actually reflects the reduction of both $\mathrm{C} \mathrm{I}$ and C II components. Consistently, the effect was found to be less pronounced in the middle of the A-K gap, where the contribution of the secondary plasma is smaller.

The O II abundance has a behavior similar to that of C II. The hydrogen abundance also exhibits a significant decrease due to electrode heating, although it was less marked in comparison with C II and O II ions. The difference may be a result of ionization processes of hydrogen ejected from the electrodes and larger portion of the fast hydrogen (relative to the C II and O II ions) arising from the charge-exchange process near the flashboard and, therefore, not affected by the electrode heating. C III 


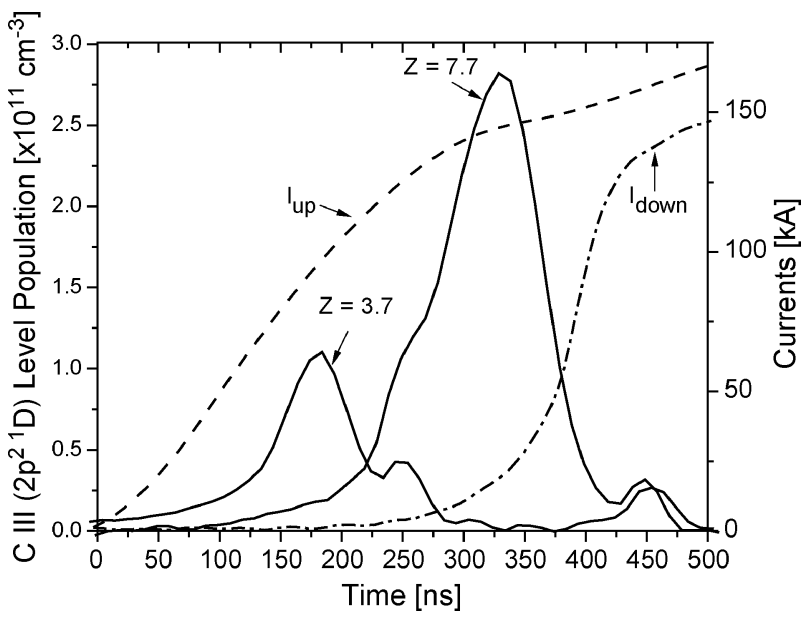

Fig. 6. Typical evolution of the C III $\left(2 \mathrm{p}^{2}{ }^{1} \mathrm{D}\right)$ level population (solid lines) for a time delay $\sim 1.7 \mu$ s observed at $z=3.7$ and $7.7 \mathrm{~cm}$ at $x=1.0 \mathrm{~cm}$ (middle of the A-K gap), integrated over the $y$-coordinate. Also shown are the upstream and downstream POS currents (dashed and dash-dotted lines, respectively).

ions are less affected by the electrode sputtering because they mainly originate from the flashboard plasma.

\section{Magnetic Field Propagation Velocity}

Employing the method described in Section IV for controlling the proton to carbon ion ratio, we have examined the effect of the plasma composition and density on the velocity of the magnetic field propagation along the plasma length (the $z$-dimension). Previous direct measurements of the magnetic field evolution by means of the Zeeman effect, have shown that the propagation of the magnetic field along the plasma is associated with a significant rise of line intensities [15], i.e., the arrival of the axially propagating magnetic field front to a specific region results in a sharp rise of light emission from that region. This finding provides a relatively simple means to measure the average velocity of the magnetic field, as demonstrated in Fig. 6, where we present the C III $\left(2 \mathrm{p}^{2}{ }^{1} \mathrm{D}\right)$ level population deduced from the line intensity observed at two different regions of the plasma $(z=3.7$ and $7.7 \mathrm{~cm})$ as a function of time. Since the appearance of the peak intensity of this line at a given axial position corresponds to the magnetic field front arrival, the distance between the two regions divided by the time difference between the two peaks gives the average magnetic field velocity. The C III-signal peak intensity observed at $z=3.7 \mathrm{~cm}$ is smaller than that observed at $z=7.7 \mathrm{~cm}$ mainly since the carbon density increases by the time the magnetic field propagates from $z=3.7 \mathrm{~cm}$ to $z=7.7 \mathrm{~cm}$. Also given in Fig. 6 is the evolution of the generator current pulse that flows through the plasma.

We have used the procedure described above to measure the average magnetic field velocity over the upstream 3.7- and $7.7-\mathrm{cm}$ parts of the plasma at $x=1.0$ and $2.2 \mathrm{~cm}$ from the cathode for various plasma compositions and densities. The measured velocities are presented in Fig. 7 as a function of the time delay between the discharge of the flashboard and the application of the generator current, where short time delays correspond to the relatively low density, proton-rich plasma, and long time delays correspond to the carbon-rich plasma,

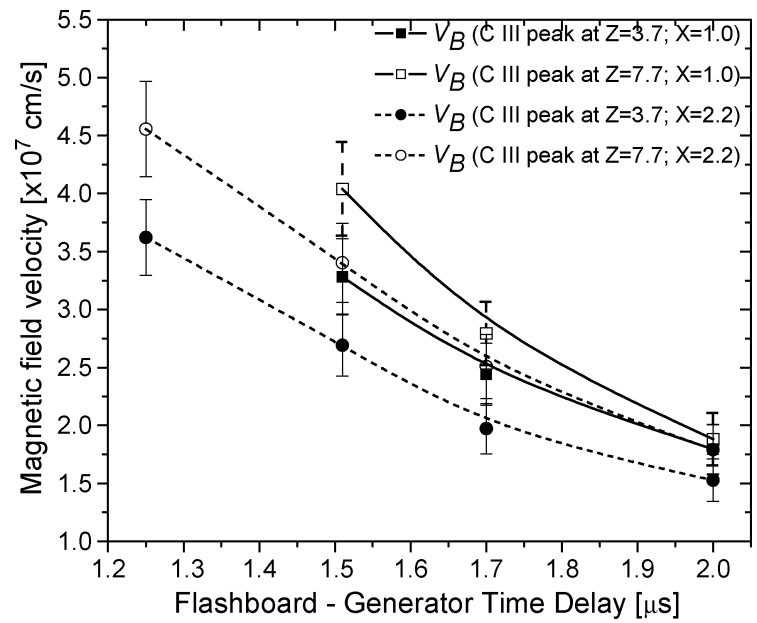

Fig. 7. Average magnetic field penetration velocity as a function of the flashboard-generator time delay obtained from the peak intensities of the $\mathrm{C}$ III line emission observed at $z=3.7$ (bold squares and circles) and $7.7 \mathrm{~cm}$ (hollow squares and circles) at distances $x=1.0$ and $2.2 \mathrm{~cm}$ from the cathode (solid and dashed lines).

as discussed in Section IV. No data is available for short time delays for $x=1 \mathrm{~cm}$ due to small number of carbon ions. We note that the use of different spectral lines for this measurement might show somewhat different velocities, depending on the details of the atomic properties of the specific transitions. However, all the lines observed yielded similar velocities (up to $25 \%$ difference) and exhibited very close trends of the velocities as a function of the flashboard-generator time delay.

It is clearly seen in Fig. 7 that the magnetic field velocity decreases with increasing time delay, i.e., with increasing fraction of carbon ions and density. As pointed out in Section III, magnetic field velocities of the order of $3 \times 10^{7} \mathrm{~cm} \cdot \mathrm{s}^{-1}$ for the present collisionless plasma cannot be explained by classical diffusion but rather by the Hall mechanism. We emphasize that the simple Hall field theory that predicts one-dimensional magnetic field penetration cannot be completely adequate for the description of the present experiment since it is only valid for cases in which the magnetic field propagates much faster than all of the plasma ions. The observed small 2-D effects [15] also contribute to the theory inapplicability. However, in the absence of a more detailed theory, it is interesting to compare qualitatively our results with the prediction of the Hall theory. According to the Hall field theory, it can be shown [16] that the characteristic velocity of the magnetic field penetration into the plasma is given by

$$
v_{\mathrm{B}}^{\text {Hall }}=\frac{c B}{8 \pi e n_{e} L}
$$

where $B$ is the magnetic field, $e$ is the electron charge, $c$ is the speed of light, and $L=\left[d \ln \left(n_{e}\right) / d x\right]^{-1}$ is the characteristic length scale of the electron density gradient. The Hall theory predicts a velocity that is proportional to the inverse of the electron density, which is consistent with the general trend seen in Fig. 7. However, the quite large error bars of the measurements do not allow to study the details of the observed monotonic decrease of the velocity with the increasing density. Taking into account the typical parameters of our experiment, $B=7 \mathrm{kG}$, 
$L=2.5 \mathrm{~cm}$, and an electron density of $n_{e}=4.5 \times 10^{14} \mathrm{~cm}^{-3}$ that corresponds to a time delay of $1.7 \mu \mathrm{s}$, (1) predicts a velocity of $1.5 \times 10^{7} \mathrm{~cm} \cdot \mathrm{s}^{-1}$. This value is lower than our measurements of $\sim 2.2 \times 10^{7} \mathrm{~cm} \cdot \mathrm{s}^{-1}$ for $1.7 \mu \mathrm{s}$, but we note that the values of the parameters we use for calculating the Hall velocity are also subjected to quite large uncertainties, particularly the electron density and density gradients that are determined with an uncertainty of $\pm 40 \%$.

In Fig. 7, it can be seen that the magnetic field penetrates the plasma faster in the middle of the A-K gap $(x=1.0 \mathrm{~cm})$ than near the anode $(x=2.2 \mathrm{~cm})$. This can be explained by the fact that the plasma near the anode is denser and contains fewer protons in comparison with the central part of the plasma.

A comparison between the average velocity measured for the $7.7-\mathrm{cm}$ part of the plasma and that measured only for the $3.7-\mathrm{cm}$-long generator side part of the plasma shows that the magnetic field velocity increases with increasing distance of propagation. This fact is attributed to the rise time of the generator current (and the magnetic field associated with it); see Fig. 6. It can be further seen that the effect of increased average velocity with increasing distance appears to diminish as the flashboard-generator time delay increases. For short time delays, the velocity at the generator-side $3.7-\mathrm{cm}$-long edge of the plasma is almost 1.5 times smaller than for the $7.7 \mathrm{~cm}$ of the plasma, whereas for large time delays, the average velocities become similar. This observation indicates that for a higher density plasma, composed of heavy constituents, the magnetic field front mainly accelerates at the generator-side edge of the plasma column and then propagates at nearly constant velocity.

\section{CONCLUSIONS}

Time-dependent 3-D-spatially-resolved observations of the magnetic field evolution and ion dynamics in POS experiments revealed a new phenomenon of ion-species separation in which the light-ion plasma is pushed ahead, whereas the heavy ion plasma lags behind the propagating magnetic piston. A detailed investigation of the ion dynamics strongly suggests that the plasma composition plays a significant role in the plasma magnetic field interaction. Motivated by these results, we have developed a method for performing experiments with controllable plasma compositions. Our measurements show that by improving the spatial separation of the constituents of the flashboard plasma source, it is possible to operate the POS in regimes in which the plasma composition varies from $\sim 90 \%$ protons to over $95 \%$ carbon ions. A drawback of this method is that the higher carbon fraction in the plasma is accompanied by a higher electron density. The control of the plasma composition is further improved by heating the electrodes to $600^{\circ} \mathrm{C}$, which significantly reduces the plasma contamination due to sputtering of species adsorbed on the electrodes. Measurements that are performed for different plasma compositions demonstrated a strong dependence of the magnetic field propagation velocity on the plasma composition and density. Further investigations are needed to determinate the density effects from those of the plasma composition. The use of the "inverse pinch" plasma source that was recently suggested and implemented in a cylindrical POS configuration in producing single-ion species plasmas with high degree of purity should be well considered [28], [29]. Calculations of the magnetic field velocities using the simple Hall field theory give values that are reasonably close to the measurements, although they appear to consistently fall within the lower limit of the error bars. In addition, the trend of the velocity dependence on the electron density, predicted by the Hall theory, is consistent with our measurements. These results show that in principle, the Hall mechanism can provide a satisfactory explanation for the magnetic field evolution, but a modified Hall theory that will take into account the plasma composition and the 2-D magnetic field penetration effects will be more adequate.

\section{ACKNOWLEDGMENT}

The authors are grateful to R. J. Commisso, H. R. Griem, S. B. Swanekamp, B. V. Weber, D. Mosher, J. W. Schumer, J. Thompson, and J. F. Drake for highly stimulating discussions. They are also indebted to P. Meiri for his skilled technical assistance.

\section{REFERENCES}

[1] J. M. Neri, J. R. Boller, P. F. Ottinger, B. V. Weber, and F. C. Young, "High-voltage, high-power operation of the plasma erosion opening switch," Appl. Phys. Lett., vol. 50, p. 1331, 1987.

[2] P. J. Goodrich, J. R. Boller, R. J. Commisso, D. D. Hinshelwood, J. C. Kellogg, and B. V. Weber, "Investigation of plasma opening switch conduction and opening mechanisms," in Proc. 8th IEEE Pulsed-Power Conf., San Diego, CA, 1991, IEEE Cat. 91CH3052-8, p. 515.

[3] D. C. Black, R. J. Commisso, P. F. Ottinger, S. B. Swanekamp, and B. V. Weber, "Experimental determination of gap scaling in a plasma opening switch," Phys. Plasmas, vol. 7, p. 3790, 2000.

[4] P. Coleman, J. Rauch, W. Rix, J. Thompson, and R. Wilson, "Recent ACE 4 Z-pinch experiments: long implosion time argon loads, uniform fill versus annular shell distributions and the Rayleigh-Taylor instability problem," in Proc. 4th Int. Conf. Dense Z-Pinches, Vancouver, BC, Canada, 1997, AIP Conference Proc. 409, p. 119.

[5] R. J. Commisso, J. P. Apruzese, D. C. Black, J. R. Boller, B. Moosman, D. Mosher, S. J. Stephanakis, B. V. Weber, and F. C. Joung, "Results of radius scaling experiments and analysis of neon $\mathrm{K}$-shell radiation data from an inductively driven Z-pinch," IEEE Trans. Plasma Sci., vol. 26, p. 1068, Aug. 1998.

[6] B. V. Weber, P. F. Ottinger, R. J. Commisso, J. R. Goyer, D. Kortbawi, J. Thompson, J. E. Rowley, P. Filios, and M. A. Babineau, "The DECADE performance assessment program," in Proc. 11th Int. Conf. High-Power Particle Beams, Prague, Czech Republic, June 1996, p. 121.

[7] B. V. Weber, D. C. Black, B. Moosman, S. J. Stephanakis, D. D. Hinshelwood, R. J. Commisso, S. B. Swanekamp, J. W. Schumer, P. F. Ottinger, J. J. Moschella, and K. Vidoli, "Comparison of POS-load dynamics using different plasma sources on HAWK," in Proc. 12th Int. Conf. High-Power Particle Beams, Haifa, Israel, 1998, IEEE Cat. no. 98EX103, p. 5.

[8] D. C. Black, J. R. Boller, R. J. Commisso, M. C. Myers, S. J. Stephanakis, and B. V. Weber, , NRL Memo. Rep. 6770-99-8392, 1999.

[9] B. V. Weber, D. D. Hinshelwood, and R. J. Commisso, "Interferometry of flashboard and cable-gun plasma opening switches on hawk," IEEE Trans. Plasma Sci., vol. 25, p. 189, Apr. 1997.

[10] Y. E. Krasik and A. Weingarten, "Energetic electron and ion beam generation in plasma opening switches," IEEE Trans. Plasma Sci., vol. 26, p. 208, Apr. 1998.

[11] B. V. Weber, R. J. Commisso, R. A. Meger, J. M. Neri, W. F. Oliphant, and P. F. Ottinger, "Current distribution in a plasma erosion opening switch,” Appl. Phys. Let., vol. 45, no. 10, pp. 1043-1045, 1984.

[12] M. Sarfaty, Y. Maron, Y. E. Krasik, A. Weingarten, R. Arad, R. Shpitalnik, and A. Fruchtman, "Spectroscopic investigations of the plasma behavior in a plasma opening switch experiment," Phys. Plasmas, vol. 2, no. 6, p. 2122, 1995.

[13] G. G. Spanjers, E. J. Yadlowsky, and R. C. Hazelton et al., "Characterization of magnetohydrodynamic effects in a plasma opening switch," $J$. Appl. Phys., vol. 77, no. 8, pp. 3657-3667, 1995. 
[14] R. Shpitalnik, A. Weingarten, K. Gomberoff, Y. Krasik, and Y. Maron, "Observations of two-dimensional magnetic field evolution in a plasma opening switch,” Phys. Plasmas, vol. 5, no. 3, p. 792, 1998.

[15] R. Arad, K. Tsigutkin, A. Fruchtman, J. D. Huba, and Y. Maron, "Observation of faster-than-diffusion magnetic field penetration into a plasma," Phys. Plasmas, vol. 10, p. 112, 2003.

[16] A. S. Kingsep, Y. V. Mokhov, and K. V. Chukbar, "The nondiffusion mechanism of magnetic-field penetration into a conductor," Sov. J. Plasma Phys., vol. 10, p. 495, 1984.

[17] A. Fruchtman, "Pentration and expulsion of magnetic-fields in plasmas due to the Hall field," Phys. Fluids B, vol. 3, p. 1908, 1991.

[18] A. V. Gordeev, A. S. Kingsep, and L. I. Rudakov, "Electron magnetohydrodynamics," Phys. Rep., vol. 243, p. 215, 1994.

[19] R. Arad, K. Tsigutkin, Y. V. Ralchenko, and Y. Maron, "Spectroscopic investigations of a dielectric-surface-discharge plasma source," Phys. Plasmas, vol. 7, p. 3797, 2000.

[20] A. Weingarten, C. Grabowski, A. Fruchtman, and Y. Maron, "The time-dependent electron density and magnetic field distribution in 70-ns plasma opening switch," in Proc. 11th Int. Conf. High-Power Particle Beams, Haifa, Israel, June 1998.

[21] A. Weingarten, R. Arad, A. Fruchtman, and Y. Maron, "Ion separation due to magnetic field penetration into a multispecies plasma," Phys. Rev. Lett., vol. 87, p. $115004,2001$.

[22] K. Tsigutkin, R. Arad, A. Fisher, and Y. Maron, "Spectroscopic study of the magnetic field, ion dynamics and electron density evolution in a plasma opening switch," in Proc. 13th Int. Conf. High-Power Particle Beams, Nagaoka, Japan, June 25-30, 2000.

[23] S. B. Swanekamp, R. J. Commisso, P. F. Ottinger, J. W. Schumer, S. D. Strasburg, B. V. Weber, Y. Maron, and R. Arad, "Species separation and field-penetration in a multi-component plasma," in AIP Conf. Proc., no. 650, 2002, pp. 455-458.

[24] R. Arad, L. Ding, and Y. Maron, "Novel gas-doping technique for local spectroscopic measurements in pulsed-power systems," Rev. Sci. Instr., vol. 69, p. 1529, 1998.

[25] R. Arad, K. Tsigutkin, Y. V. Ralchenko, A. Fruchtman, and Y. Maron, "Investigation of the ion dynamics in a multi-species plasma under pulsed magnetic fields," Phys. Plasmas, to be published.

[26] R. Arad, Ph.D. dissertation, Weizmann Inst. Science, Rehovot, Israel, 2002.

[27] J. D. Huba, "Hall magnetohydrodynamics in-space and laboratory plasmas," Phys. Plasmas, vol. 2, p. 2504, 1995.

[28] J. J. Moschella, R. C. Hazelton, C. Vidoli, E. J. Yadlowsky, B. V. Weber, D. C. Black, D. D. Hinshelwood, B. Moosmann, and S. J. Stephanakis, "POS experiments on HAWK using an inverse pinch, gas plasma source," in Proc. 11th Int. Conf. High-Power Particle Beams, Haifa, Israel, June 1998, p. 305.

[29] J. J. Moschella, R. C. Hazelton, C. Vidoli, and E. J. Yadlowsky, “An inverse pinch plasma source for plasma opening switches," IEEE Trans. Plasma Sci., vol. 28, pp. 2247-2255, Dec. 2000.

[30] J. R. Goyer, D. Kortbawi, F. K. Childers, P. S. Sincerny, B. V. Weber, P. F. Ottinger, R. J. Commisso, J. Thompson, and M. A. Babineau, "Plasma opening switch research for DECADE," IEEE Trans. Plasma Sci., vol. 25, pp. 176-188, Apr. 1997.

[31] Russian Phys. J., vol. 40, p. 1154, 1997.

[32] K. Tsigutkin, R. Arad, A. Fisher, and Y. Maron, "Spectroscopic study of the magnetic field, ion dynamics and electron density evolution in a plasma opening switch," in Proc. 13th Int. Conf. High-Power Particle Beams, Nagaoka, Japan, 2000, p. 111.
D. Osin, photograph and biography not available at time of publication.

R. Doron, photograph and biography not available at time of publication.

R. Arad, photograph and biography not available at time of publication.

K. Tsigutkin, photograph and biography not available at time of publication.

A. Starobinets, photograph and biography not available at time of publication

V. Bernshtam, photograph and biography not available at time of publication.

A. Fisher, photograph and biography not available at time of publication.

A. Fruchtman, photograph and biography not available at time of publication.

Y. Maron, photograph and biography not available at time of publication.

A. Tauschwitz, photograph and biography not available at time of publication 